\title{
Comparação de simulações dinâmicas de máquinas síncronas entre o ANATEM - CEPEL e o modelo de dois eixos
}

\author{
Anderson de Sousa Duarte ${ }^{1}$, Adriano Aron Freitas de Moura ${ }^{2}$, Ailson Pereira de Moura ${ }^{3}$, Idalmir de \\ Souza Queiroz Júnior ${ }^{4}$, Olympio Cipriano da Silva Filho ${ }^{5}$, Marcelo Roberto Bastos Guerra Vale ${ }^{6}$ \\ [1] Universidade Federal Rural do Semi-Árido (UFERSA) \\ [2] Universidade Federal Rural do Semi-Árido (UFERSA); adrianoaron@hotmail.com \\ [3] Universidade Federal do Ceará (UFC) \\ ${ }^{[4]}$ Universidade Federal Rural do Semi-Árido (UFERSA) \\ ${ }^{[5]}$ Universidade Federal Rural do Semi-Árido (UFERSA) \\ ${ }^{[6]}$ Universidade Federal Rural do Semi-Árido (UFERSA)
}

Recebido: 23/04/2020;

Aceito: $27 / 07 / 2020$;

Publicado: 11/09/2020.

Resumo: A análise de estabilidade transitória para sistemas elétricos de potência é essencial para o correto funcionamento do sistema. Deste modo, devido a confiabilidade e eficiência, o uso de ferramentas computacionais tem sido bastante utilizado a fim de auxiliar nos estudos e na busca de soluções que envolvem a análise de estabilidade. O número de distúrbios que podem afetar um sistema elétrico de potência é muito grande, sendo que o grau de seriedade desses distúrbios está relacionado a complexidade do arranjo e das malhas de controle. Com boa aproximação, será utilizado o modelo de dois eixos da máquina síncrona conectado a um barramento infinito, o problema será implementado na ferramenta do Simulink ${ }^{\circ}$ do MATLAB $®$ e após a simulação terá seus resultados comparados com resultados obtidos com o ANATEM.

Palavras-chave: Anatem; Modelo de dois eixos; Estabilidade, Máquina de polos salientes.

Abstract: Transient stability analysis for electrical power systems is essential for the correct functioning of the system. Thus, due to reliability and efficiency, the use of computational tools has been widely used in order to assist in studies and in the search for solutions that involve stability analysis. The number of disturbances that can affect an electrical power system is very large, and the degree of seriousness of these disorders is related to the complexity of the arrangement and the control meshes. With good approximation, the two-axis model of the synchronous machine connected to an infinite bus will be used, the problem will be implemented in the Simulink tool ${ }^{\circledR}$ of MATLAB ${ }^{\circledR}$ and after the simulation will have its results compared with results obtained with ANATEM.

Keywords: Anatem; two axes model; Stability, salient pole machine. 
A estabilidade do sistema de potência pode ser definida como a propriedade do sistema que permite às máquinas síncronas desse sistema responder a um distúrbio, a partir de uma condição normal de operação, de tal maneira a retomarem uma condição de operação novamente normal [1].

O presente trabalho, pretende fazer uma análise comparativa entre os resultados obtidos para um problema proposto no software Análise de transitórios eletromecânico (ANATEM), com os resultados obtidos no Simulink ${ }^{\circledR}$ do MATLAB ${ }^{\circ}$. Visto que, atualmente vem se tornando cada vez mais necessário o uso de ferramentas computacionais para se fazer análises de sistemas de potência.

Assim sendo, a análise de transitórios eletromecânicos para sistemas elétricos de potência é essencial para o correto funcionamento dos sistemas de potência, o estudo do comportamento transitório das maquinas síncronas envolve tanto fenômeno elétrico, que relaciona fluxos e correntes, como fenômeno mecânico, que descreve as variações da velocidade do eixo e do ângulo do rotor [2].

Com o intuito de auxiliar nas estratégias e tendo em vista que a análise de sistemas de potência é muita complexa tem-se utilizado constantemente ferramentas computacionais para simulação do comportamento do sistema elétrico de potência (SEP), é uma ferramenta importante para o planejamento, análise de estabilidade. No SEP, o uso de ferramentas computacionais é necessário para garantir um sistema confiável e seguro. O ANATEM é uma aplicação computacional para a realização de estabilidade à frequência fundamental tanto na operação como no SEP. Inicialmente serão apresentadas de forma bem reduzida as principais relações da máquina síncrona, e em seguida será apresentada a transformação dq0, sob a qual se desenvolverá a teoria de máquina síncrona de polos salientes. No artigo é desenvolvido uma situação problema para uma máquina síncrona de polos salientes conectada a um barramento infinito. O modelo de dois eixos apresentado no decorrer do artigo será modelado no Simulink ${ }^{\circledR}$, de forma que os resultados obtidos estejam em conformidade aos resultados encontrados no ANATEM.

\section{ESTABILIDADE DE SISTEMAS ELÉTRICOS DE POTÊNCIA}

A tendência de um sistema de potência é desenvolver forças restauradoras, iguais ou maiores que as forças perturbadoras, para que se possa manter o estado de equilíbrio da máquina é conhecida como estabilidade. Se as forças que tendem a manter as máquinas sincronizadas umas com as outras são iguais as forças perturbadoras são ditas que o sistema permanece estável, permanecendo em sincronismo [2].

O problema da estabilidade está relacionado ao comportamento das máquinas síncronas após uma perturbação, por conveniência da análise os problemas de estabilidade são geralmente divididos em duas categorias principais, estabilidade em estado estacionário e estabilidade transitória. A estabilidade em estado estacionário refere-se à capacidade do sistema de energia em recuperar o sincronismo após pequenas e lentas distorções, como mudanças graduais de energia. Uma extensão da estabilidade em estado estacionário é conhecida como estabilidade dinâmica. A estabilidade dinâmica preocupa-se com pequenas perturbações que duram muito tempo com a inclusão de dispositivos de controle automático. Estudos de estabilidade transitória lidam com os efeitos de distúrbios grandes e repentinos, como a ocorrência de uma falha, a interrupção repentina da linha ou a aplicação ou remoção repentina de cargas. São necessários estudos de estabilidade transitória para garantir que o sistema possa suportar a condição transitória após uma grande perturbação. Frequentemente, esses estudos são realizados quando se quer realizar novas gerações de energia e transmissão planejadas [2].

\section{1. Ângulo Crítico de Abertura}


Considerando um sistema composto, pela máquina conectada à uma barra infinita e para uma dada falta $\mathrm{e}$ combinação de chaveamento, o ângulo crítico de eliminação da falta é o máximo ângulo para eliminação do defeito sem que o sistema perca a estabilidade.

A análise considera primeiramente a Figura 1, em que são representadas as curvas potência-ângulo de um sistema para as situações de antes, durante e pós-falta, supondo-se que a abertura da falta ocorre no instante em que $\delta=\delta c$. Sendo assim, é possível notar que podemos mover o ponto f para a direita, acarretando assim um ângulo de abertura maior, ou seja, maior tempo de abertura do defeito. O ângulo de abertura deste caso não é crítico.

Por meio das considerações acima, segundo [3], para qualquer carregamento inicial dado, existe um ângulo crítico de abertura $\delta$ crit além do qual não será mais possível obter uma área A2 igual à área A1. Para ângulos de abertura reais do defeito menores que $\delta c r i t$ o sistema será estável. Se, por outro lado $\delta c>\delta c r i t$, o sistema é instável.

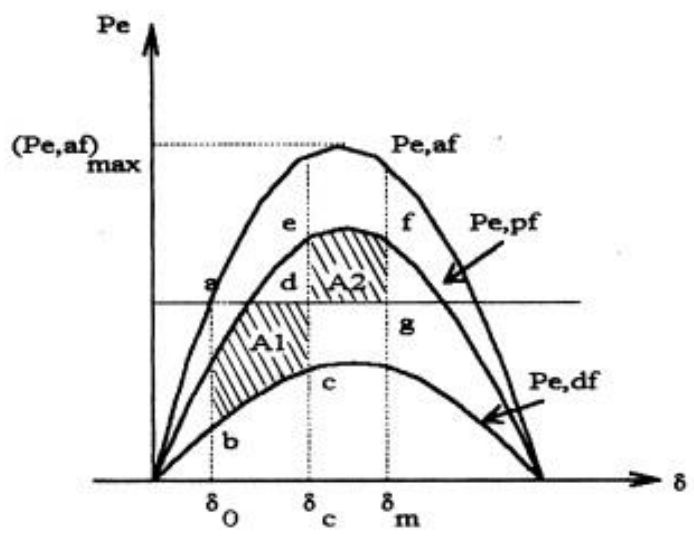

FIGURA 1. Curvas potência-ângulo supondo abertura da linha após a falta [3].

\section{MÁQUINA DE POLOS SALIENTES}

Visto que em uma máquina de polos salientes, diferentemente das máquinas de rotor liso existe um caminho preferencial de fluxo determinado pela saliência dos polos do rotor. A permeância magnética será maior ao longo do eixo polar e menor no eixo entre os polos, também chamada de região interpolar. Devido a essa diferença, é possível concluir que a indutância mútua de estator de uma máquina síncrona de polos salientes varia em função da posição angular do rotor, e consequentemente, em função do tempo. Consequentemente, há um problema na hora do desenvolvimento do modelo matemático pois a distribuição de fluxo não é mais uma distribuição senoidal ao longo do entreferro devido ao surgimento de componentes harmônicas provenientes da saliência dos polos. Outro fator são os harmônicos espaciais existentes ao longo da máquina, em termos construtivos, a máquina é feita de forma e reduzir a influência desses harmônicos nas ondas de fluxo concatenado pelos enrolamentos, porém eles não são totalmente eliminados podendo ser observados nas ondas de fluxo magnético [4].

\subsection{Transformada de Park}

Na engenharia sempre que encontramos problemas complexos tentamos simplificado para modelos de fácil manuseio sem descaracterizar de suas características originais, a transformada de Park serve justamente par isso tentar facilitar a interpretação e modelagem de uma máquina de polos salientes devido aos problemas que surgem das saliências dos polos. 
A conversão do sistema de referência é feita através da transformada de Park $[\boldsymbol{P}(\theta r)]$ dada pela equação (1). Basicamente, esta matriz de transformação projeta as grandezas de um sistema trifásico em um sistema bifásico fictício, tendo como referencial o eixo direto e o eixo em quadratura do rotor [5].

$$
P\left(\theta_{r}\right)=\left[\begin{array}{ccc}
\cos \left(-\theta_{r}\right) & \cos \left(-\theta_{r}+\frac{2 \pi}{3}\right) & \cos \left(-\theta_{r}-\frac{2 \pi}{3}\right) \\
\operatorname{sen}\left(-\theta_{r}\right) & \operatorname{sen}\left(-\theta_{r}+\frac{2 \pi}{3}\right) & \operatorname{sen}\left(-\theta_{r}-\frac{2 \pi}{3}\right) \\
\frac{1}{2} & \frac{1}{2} & \frac{1}{2}
\end{array}\right]
$$

Esse conceito fica mais claro com a ilustração da Figura 2. A grande vantagem dessa transformação é eliminar a dependência que as indutâncias possuem da posição angular do rotor [5].

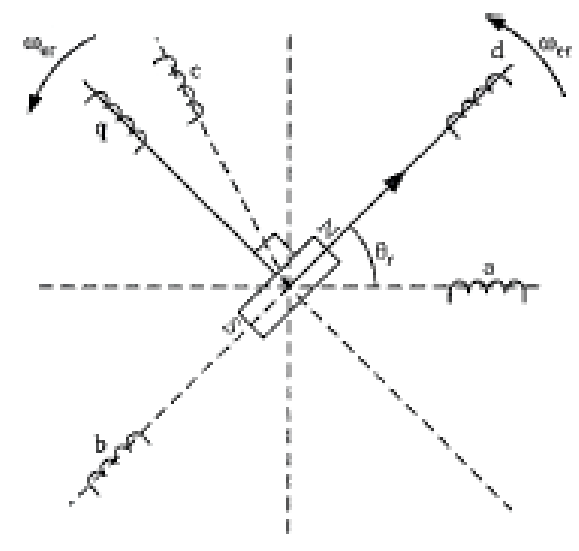

FIGURA 2. Transformação do sistema trifásico no sistema d-q [5].

A transformada de Park muda o sistema de referência do estator para o rotor. Para que a transformada exista, é necessário introduzir grandezas de sequência zero na modelagem, que, no caso das correntes trifásicas, é igual à $(i a+i b+i c) / 3$.

\subsection{Análise de Transitórios Eletromecânicos (ANATEM)}

O Programa de estabilidade transitória utilizado foi o ANATEM, desenvolvido pelo Centro de Pesquisas Energia Elétrica (CEPEL), que tem como objetivo simular o comportamento dinâmico de sistemas de potência quando submetido a perturbações.

Sendo possível, realizar simulações no domínio do tempo de SEP de grande porte, realizando a análise não linear da estabilidade eletromecânica. Com seu uso, é possível determinar a estabilidade dos sistemas modelados, conhecendo sua resposta dinâmica após a ocorrência de perturbações.

O programa proporciona a resposta frente uma sequência de perturbações, seja ela de pequena ou grande magnitude, seguida por operações de abertura e religamento de circuitos de transmissão. O desligamento de outros elementos também pode ser considerado. Os módulos que compõem o ANATEM foram codificados em FORTRAN e a capacidade do programa é definida através de um arquivo de parâmetros que facilita o seu redimensionamento de acordo com as necessidades e instalações computacionais especificas de cada usuário [6].

Ao ser instalado o ANATEM, é possível instalar o programa auxiliar Plot do CEPEL, por que apresenta os dados de plotagem especificados no arquivo de simulação logo após a execução da simulação, abrindo 
automaticamente. Os gráficos apresentados nas figuras deste artigo são provenientes do Plot.

\section{MODELO DE DOIS EIXOS}

Diante de outras restrições de terminal possam ser especificadas, o barramento infinito é o mais amplamente utilizado para análise de uma máquina, em parte porque é tradicional o estudo de um único gerador com toda a rede restante como uma fonte de tensão e impedância equivalente de Thevenin. Tais equivalentes são claramente válidos, apenas para algumas condições de estado estacionário, outros usos de modelos de barramento infinito para máquinas surgiram recentemente como mecanismos para evitar problemas associados para o ângulo de referência e velocidade de estado estacionário [4]. O modelo de ordem reduzida apresentado na figura 3 contêm a dinâmica do enrolamento $\boldsymbol{\Psi}_{\mathbf{1} \boldsymbol{d}}$ e $\boldsymbol{\Psi}_{\mathbf{2 q}}$, se $\mathbf{T}_{\boldsymbol{d} \mathbf{0}}^{\prime}$ e $\mathbf{T}_{\boldsymbol{q} \mathbf{0}}^{\prime}$ são suficientemente pequenos, existe um enrolamento amortecido integral entre esses estados dinâmicos.

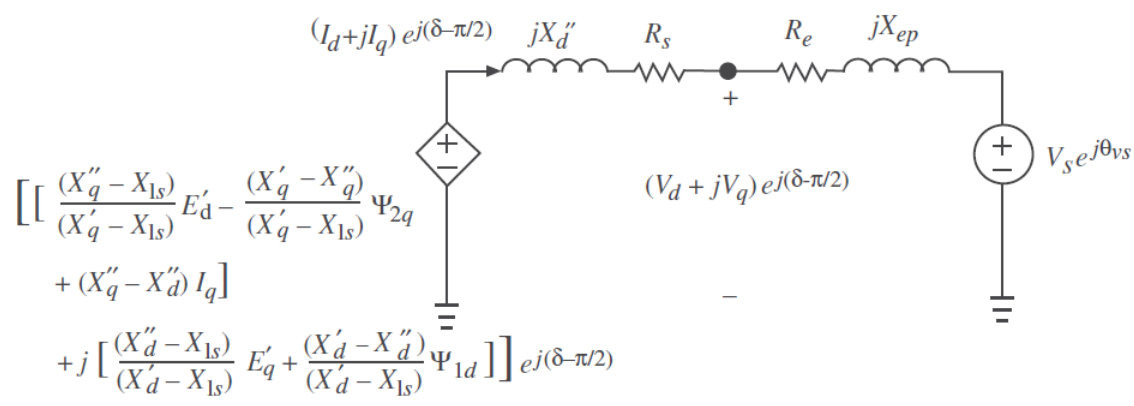

FIGURA 3. Circuito dinâmico sub-trânsitório da máquina síncrona. [7]

O modelo de dois eixos apresentado na figura 4 é obtido através de simplificações do [7], eliminando a dinâmica do enrolamento do estator.

Onde:

$\boldsymbol{E}_{\boldsymbol{d}}{ }_{\boldsymbol{d}}$ : Tensão transitória de eixo direto

$\boldsymbol{V}_{\boldsymbol{S}}$ : Tensão nos terminais da máquina

$\boldsymbol{X}_{\boldsymbol{e p}}$ : Reatância dinâmica do estator

$\boldsymbol{R}_{\boldsymbol{e}}$ : Resistência no terminal da máquina

$\boldsymbol{R}_{\boldsymbol{s}}$ : Resistencia de dispersão da armadura

$\boldsymbol{X}_{\boldsymbol{q}}{ }_{\boldsymbol{q}}$ : Reatância transitória de eixo de quadratura

$\boldsymbol{X}_{\boldsymbol{d}}^{\prime}$ : Reatância transitória de eixo de quadratura

$\boldsymbol{X}^{\prime \prime}{ }_{\boldsymbol{q}}$ : Reatância subtransitória de eixo de quadratura

$\boldsymbol{X}_{\boldsymbol{d}}{ }_{\boldsymbol{d}}$ Reatância subtransitória de eixo direto

$\boldsymbol{X}_{\mathbf{1 s}}$ : Reatância no terminal da máquina

$\boldsymbol{\Psi}_{1 d}$ : Fluxo concatenado do enrolamento 1 amortecido de eixo direto

$\Psi_{2 q}$ : Fluxo concatenado do enrolamento 2 amortecido de eixo de quadratura

$\boldsymbol{V}_{\boldsymbol{d}}$ : Tensão de eixo direto

$\boldsymbol{V}_{\boldsymbol{d}}$ : Tensão de eixo de quadratura 


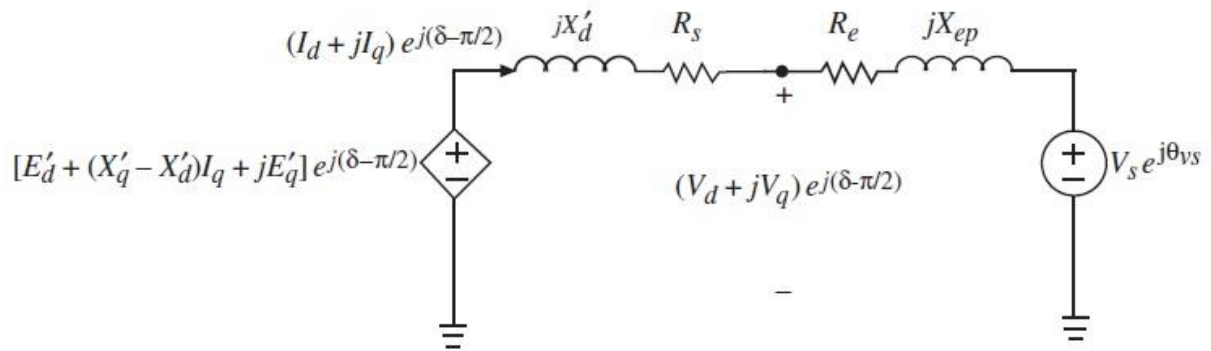

FIGURA 4. Modelo de máquina síncrona de dois eixos. [7]

\section{DADOS DO SISTEMA}

Com o propósito, de serem feitas as análises levando em consideração a máquina síncrona de 1000MW ligada a uma barra infinita através de uma reatância 0,075 ou 7,5\%. A máquina em regime, permanente entrega $1 \mathrm{pu}$ de potência ativa e 0,45 pu de potència reativa. O ângulo da barra abre 48,6 graus, o que pode ser constatado com auxilio de um programa de cálculo de fluxo de carga. Os dados maquina em pu e constantes de tempo e $\mathrm{H}$ em segundos são apresentados na tabela 1 que foram retirados do prórprio arquivo do ANATEM.

TABela 1. Dados da Máquina. (Autoria própria)

\begin{tabular}{ccccccccccc}
\hline $\boldsymbol{L}_{\boldsymbol{d}}$ & $\boldsymbol{L}_{\boldsymbol{q}}$ & $\boldsymbol{L}_{\boldsymbol{d}}^{\prime}$ & $\boldsymbol{L}_{\boldsymbol{q}}^{\prime}$ & $\boldsymbol{L}_{\boldsymbol{d}}^{\prime \prime}$ & $\boldsymbol{L}_{\boldsymbol{l}}$ & $\boldsymbol{T}_{\boldsymbol{d}}^{\prime}$ & $\boldsymbol{T}_{\boldsymbol{q}}^{\prime}$ & $\boldsymbol{T}^{\prime \prime}{ }_{\boldsymbol{d}}$ & $\boldsymbol{T}^{\prime \prime}{ }_{\boldsymbol{q}}$ & $H$ \\
\hline 1,138 & 0,681 & 0,35 & 0,35 & 0,288 & 0,0 & 5,6 & 0,15 & 0,08 & 0,15 & 4,938 \\
\hline \multicolumn{10}{c}{ Fonte: Autoria própria, 2019. }
\end{tabular}

Onde:

$\boldsymbol{L}_{\boldsymbol{d}}$ : Indutância de eixo direto

$\boldsymbol{L}_{\boldsymbol{q}}$ : Indutância de eixo de quadratura

$\boldsymbol{L}_{\boldsymbol{d}}{ }_{\boldsymbol{d}}$ : Indutância transitória eixo direto

$\boldsymbol{L}_{\boldsymbol{q}}^{\prime}$ : Indutância transitória eixo de quadratura

$\boldsymbol{L}_{\boldsymbol{d}}{ }_{\boldsymbol{d}}$ : Indutância subtransitória eixo direto

$\boldsymbol{L}^{\prime \prime}{ }_{q}$ : Indutância subtransitória eixo de quadrature

$\boldsymbol{L}_{\boldsymbol{l}}$ : Indutância de dispersão da armadura

$\boldsymbol{T}_{\boldsymbol{d}}{ }_{\boldsymbol{d}}$ : Constante de tempo transitória de eixo direto

$\boldsymbol{T}^{\prime}{ }_{\boldsymbol{q}}$ : Constante de tempo transitória de eixo de quadratura

$\boldsymbol{T}^{\prime \prime}{ }_{\boldsymbol{d}}$ : Constante de tempo subtransitória de eixo direto

$\boldsymbol{T}^{\prime \prime}{ }_{\boldsymbol{q}}$ : Constante de tempo subtransitória de eixo de quadratura

$H$ : Constante de inércia da máquina

\section{MODELAGEM ANATEM}

A Tabela 2 tem a finalidade de exibir os comandos utilizados no ANATEM apresentados na Figura 3. 
TABELA 2. Códigos utilizados no ANATEM. (Autoria prórpia)

\begin{tabular}{|c|c|}
\hline Cатро & Descrição \\
\hline $\begin{array}{l}\text { Número do modelo } \\
\text { gerador }\end{array}$ & $\begin{array}{l}\text { Número de identificação do modelo do gerador, como definido no campo Número } \\
\text { do código de execução DMDG. O modelo utilizado é o MD01 que é modelo de } \\
\text { barra infinita, definido com tensão constante atrás da reatância transitória e o } \\
\text { MD03 modelo de rotor liso com enrolamento de campo e três enrolamentos } \\
\text { amortecedor, sendo um no eixo direto e um no eixo em quadratura. }\end{array}$ \\
\hline $\begin{array}{l}\text { Associação do } \\
\text { modelo da máquina }\end{array}$ & $\begin{array}{l}\text { O código de execução DMAQ associação do modelo do gerador definido no } \\
\text { código DMDG como a barra de geração do sistema. }\end{array}$ \\
\hline Tipo de Evento & $\begin{array}{l}\text { Com o uso do código DEVT é possível realizar inúmeras possibilidades de } \\
\text { eventos. No caso em questão um curto circuito franco na barra durante } 1 \text { segundo. }\end{array}$ \\
\hline Curvas de Saturação & $\begin{array}{c}\text { No código DCST, as curvas de saturação são referenciadas para o modelo do } \\
\text { gerador. }\end{array}$ \\
\hline Variáveis & As variáveis expostas na tabela 1 são dispostas no código DPLT. \\
\hline Tempo da interação & $\begin{array}{l}\text { Utilizando o código DSIM, foi definido o tempo máximo de simulação como 30s } \\
\text { com passo de interação de } 0,005 \text { e saída de plotagem de } 1 \text { em } 1 \text { ponto. }\end{array}$ \\
\hline
\end{tabular}

Fonte: Autoria própria, 2019.

Em conformidade com o problema descrito, os dados foram modelados no ANATEM, de acordo com a figura 5 onde está sendo mostrado todo o código executado.

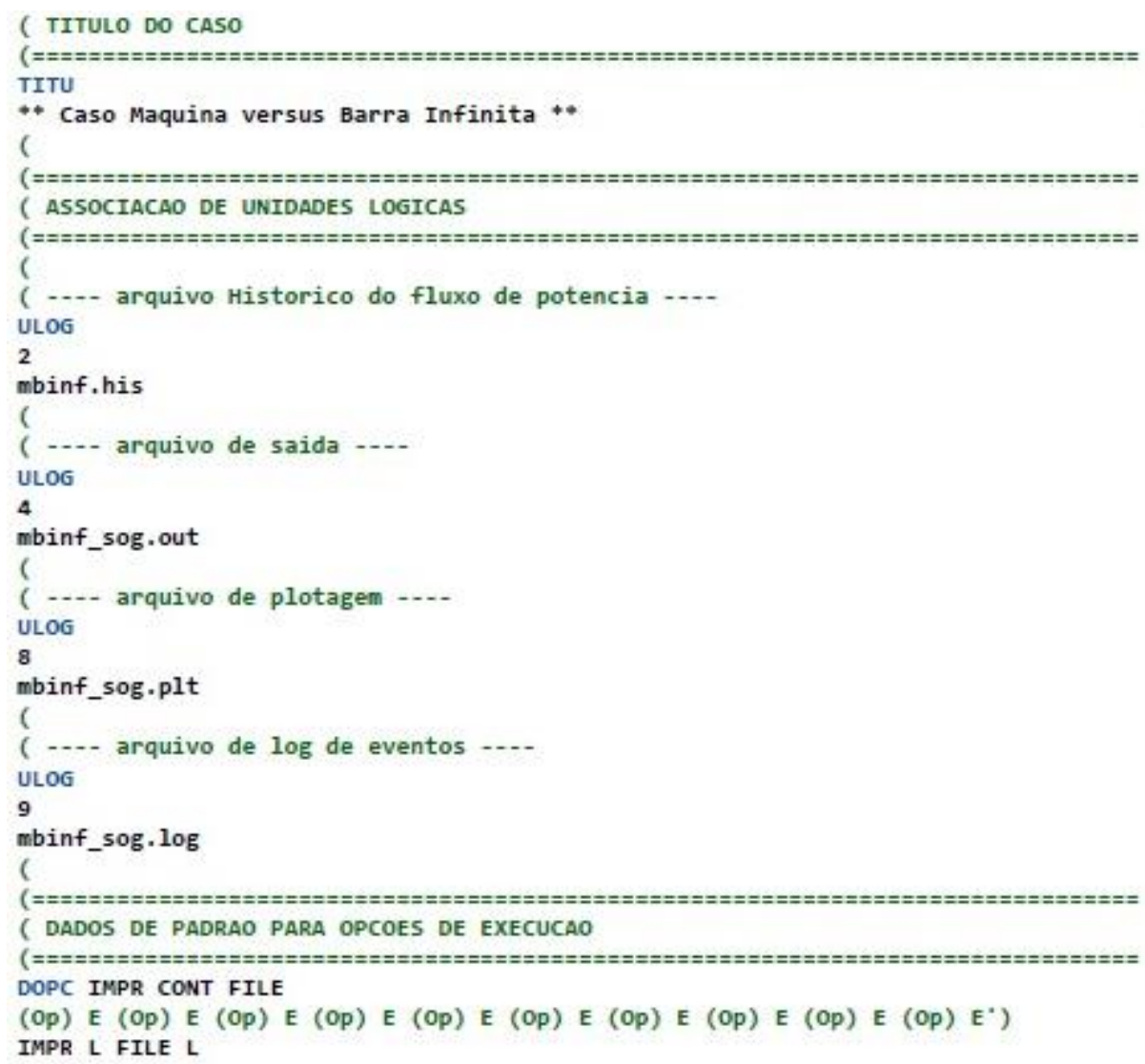




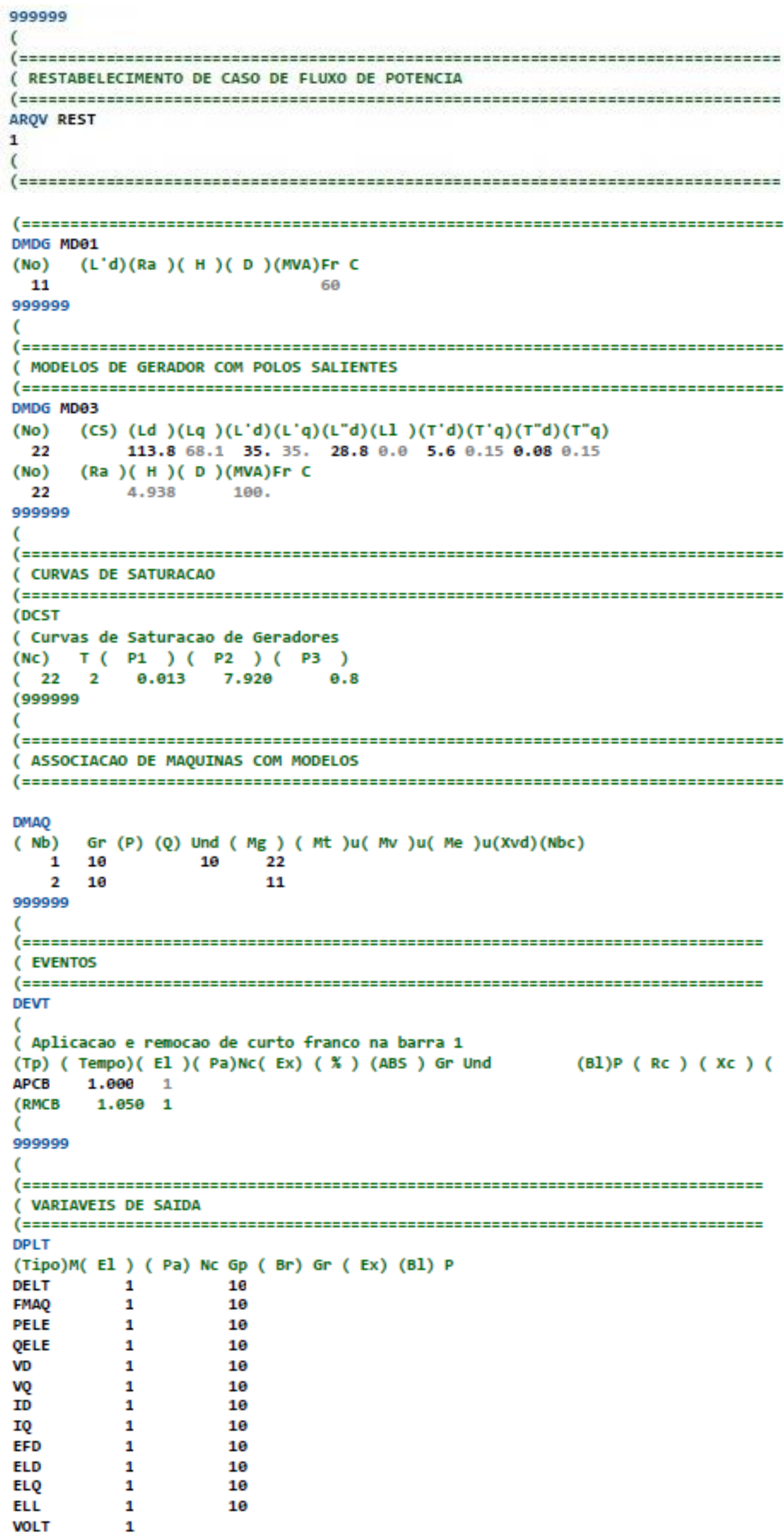




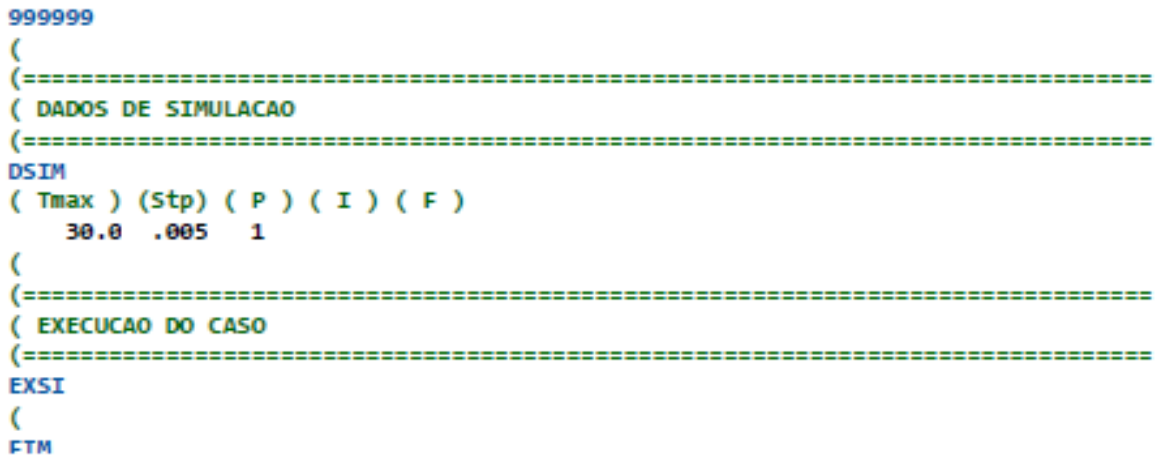

FIGURA 5. Código na interface do ANATEM (Autoria própria).

\subsection{Modelagem de Dois Eixos}

As equações abaixo são retiradas após à analise do fluxo de carga do sistema, para o modelo de dois eixos e são implementadas na ferramenta simulink®.

Inicialmente são calculados as condições iniciais da máquina síncrona a partir do estudo de fluxo de caraga realizado no ANAREDE.

$$
\begin{gathered}
\mathrm{E}^{\prime}{ }_{\mathrm{d}}=-0,35 \mathrm{I}_{\mathrm{q}}+\cos \left(138,6^{\circ}-\delta\right) \\
\mathrm{E}^{\prime}{ }_{\mathrm{q}}=0,35 \mathrm{I}_{\mathrm{d}}+\cos \left(138,6^{\circ}-\delta\right)
\end{gathered}
$$

As equações (2) e (3) representam as equações da tensão interna transitória de eixo de quadratura e a tensão interna transitória de eixo direto, elas devem ser montadas no sistema de malha de controle na ferramenta computacional junto com a equação de oscilação do ângulo do rotor da máquina síncrona.

No instante que ocorre a falta, temos que:

$$
\mathrm{V}_{\mathrm{d}}+\mathrm{j} \mathrm{V}_{\mathrm{q}}=0+\mathrm{j} 0
$$

As reatâncias a serem usadas nesse período são, a subtransitória de eixo direto e a subtransitória de eixo de quadratura, usando uma aproximação temos que:

$$
X^{\prime \prime}{ }_{q}=X_{q}-X_{q}^{2}=0,681-0,681^{2}=0,217
$$

Então para as equações do período de falta temos:

$$
\begin{gathered}
I_{d}=\frac{j E^{\prime} q}{j 0,288}=\frac{E_{q}^{\prime}}{0,288} \\
j I_{q}=\frac{-j E d}{0,217}
\end{gathered}
$$

Logo:

$$
I_{q}=\frac{-E_{d}}{0,217}
$$

A equação (9) representa a oscilação do ângulo da máquina durante o evento a ser observado.

$$
\frac{24,938}{377} \frac{\mathrm{d} \omega}{\mathrm{dt}}=1-\mathrm{E}_{\mathrm{d}}^{\prime} \mathrm{I}_{\mathrm{d}}-\mathrm{E}^{\prime}{ }_{\mathrm{q}} \mathrm{I}_{\mathrm{q}}
$$

Na figura 6 é mostrado a modelagem do problema usando o Simulink®, que é uma ferramenta de simulação integrada ao software MATLAB ${ }^{\circledR}$, que permite modelar, simular e analisar sistemas dinâmicos. Nesse sentido uma grande variedade de sistemas pode ser projetada e testada, nomeadamente sistemas de 
controle, sistemas de processamento de sinal, sistemas de comunicação, sistemas lógicos, entre muitos outros [8].

O Matlab® e o simulink® são usados convenientemente para simular o sistema, e o modelo é estendido aos sistemas de múltiplas máquinas. A estabilidade transitória usando critérios de área é discutido e o resultado é representado graficamente [9].

As equações diferenciais acima são montadas. Nos dois ganhos onde estão ligados 2 scopes são representadas as equações das correntes de eixo direto e quadratura. A parte superior representa a equação da tensão interna transitória de eixo em quadratura. A parte do meio representa a equação da tensão interna transitória de eixo direto, enquanto que a parte de baixo representa a equação de oscilação do ângulo do rotor da máquina síncrona.

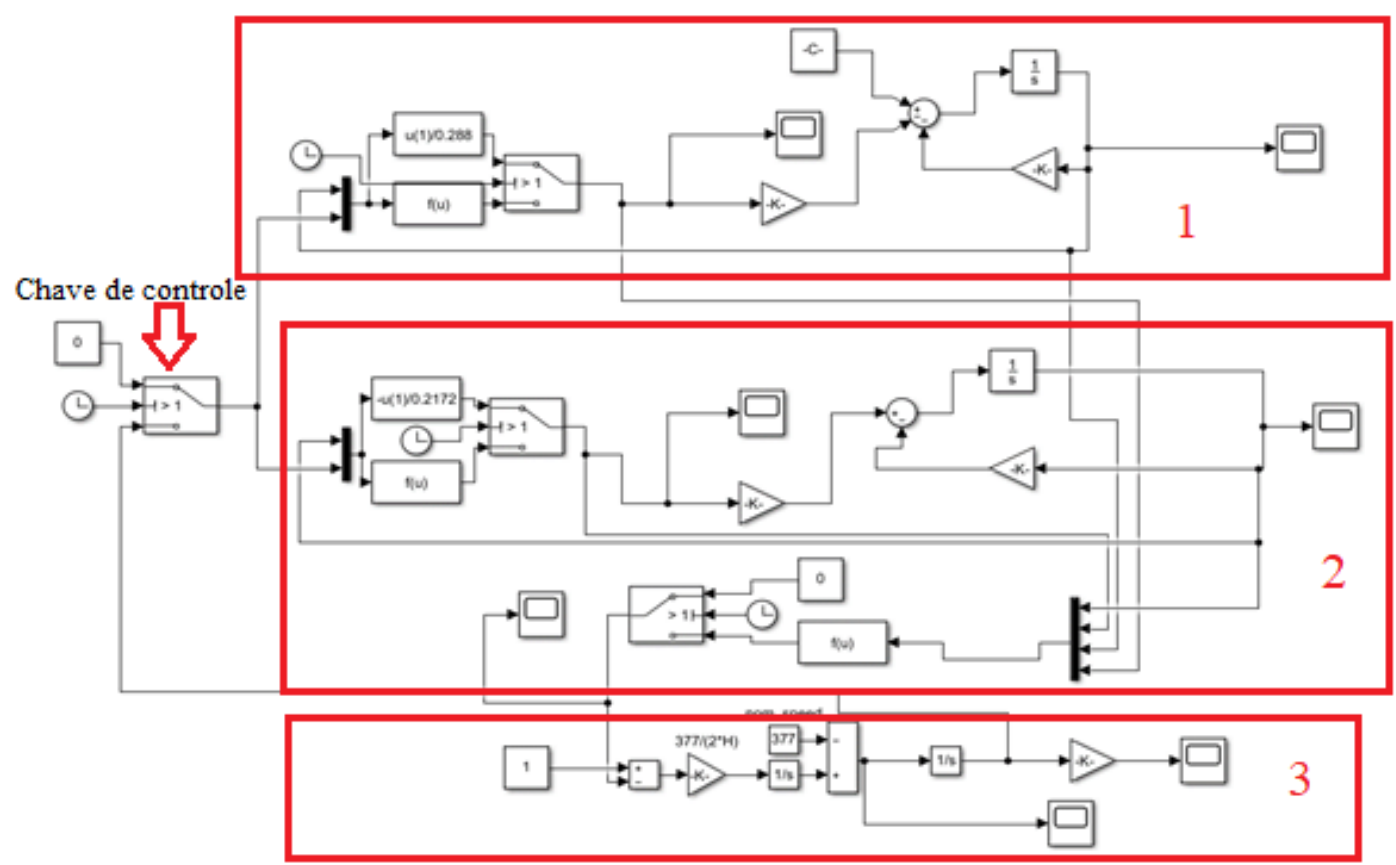

FIGURA 6. Implementação do Modelo de dois eixos no Simulink® (Autoria própria)

Para facilitar o entendimento sobre sobre a implementação das equações de controle no Simulink®, o diagrama de blocos da Figura 6 foi dividido em três sessões sendo as sessões 1 e 2 referentes a utilização das euqações das tensões e correntes de eixo direto e quadratura, na sessão 3 ficou implementada a equação (9) sendo a equação responsavel pela oscilação angular da máquina, que vai alimentar a chave que faz o controle do Sistema antes de e depois da falta.

\section{RESUlTADOS E DISCUSSÃO}

De acordo com as simulações, no ANATEM foram inseridos códigos de execução nas linhas de comando, os gráficos foram plotados com a ajuda da ferramenta Plot Cepel exibidos lado a lado com os gráficos obtidos utilizando o modelo de dois eixos através do Simulink®. Para todas as figuras onde se tem as comparações entre os gráficos, o gráfico a esquerda representa o resultado no ANATEM e o gráfico a direita representa o resultado obtido no Simulink®.

Como pode ser visto na Figura 7, dispomos do resultado das simulações para a corrente no eixo de quadratura, a corrente se mantém constante até o instante de 1 segundo que é o momento que sofre a falta ocorrendo uma inversão da polaridade encontrado a estabilidade quando seu valor permanece em zero. 

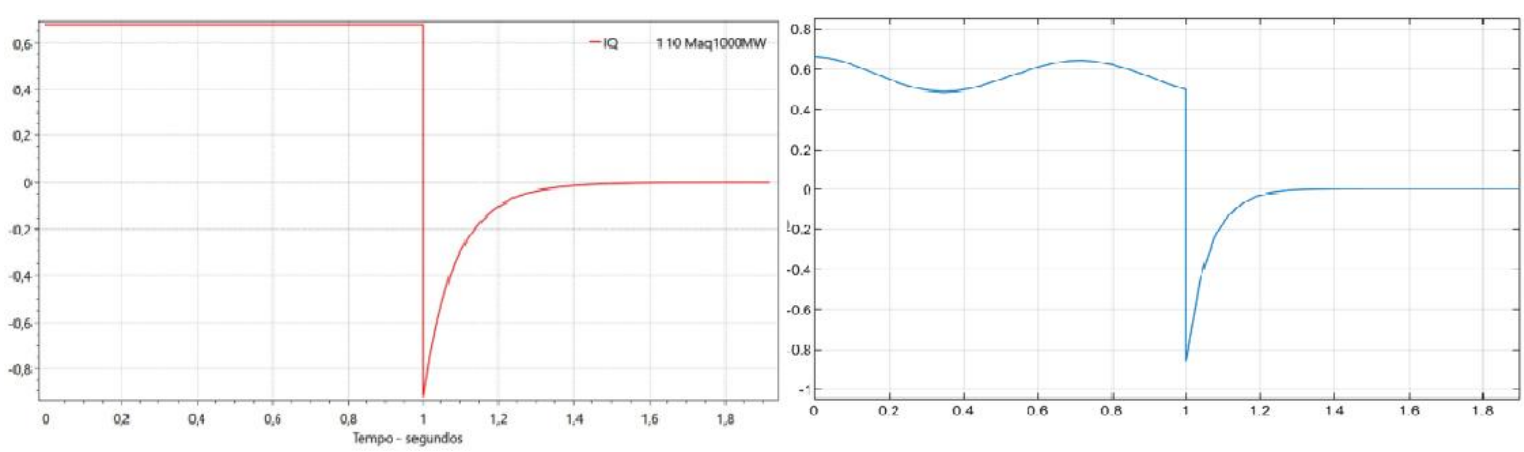

FIGURA 7. Corrente de eixo de quadratura. (Autoria própria)

Na figura 8 a corrente no eixo direto apresenta um comportamento semelhante ao comportamento da corrente no eixo de quadratura, a corrente sofre um elevado aumento e tende a diminuir o valor posteriormente, isso se deve à polaridade que pode ser visto nas equações 6 e 7.
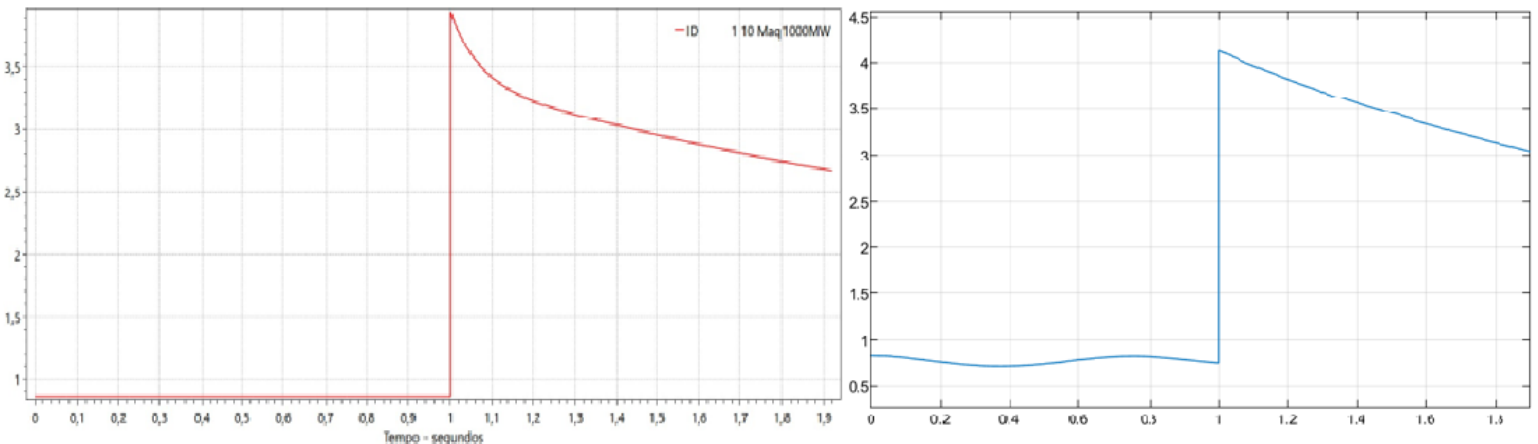

FIGURA 8. Corrente de eixo direto. (Autoria própria)

Na figura 9 após a falta a tensão no eixo de quadratura tende a zero, devido ao comportamento da corrente de quadratura que também tende a zero, a máquina tende a buscar as condições iniciais de estabilidade.
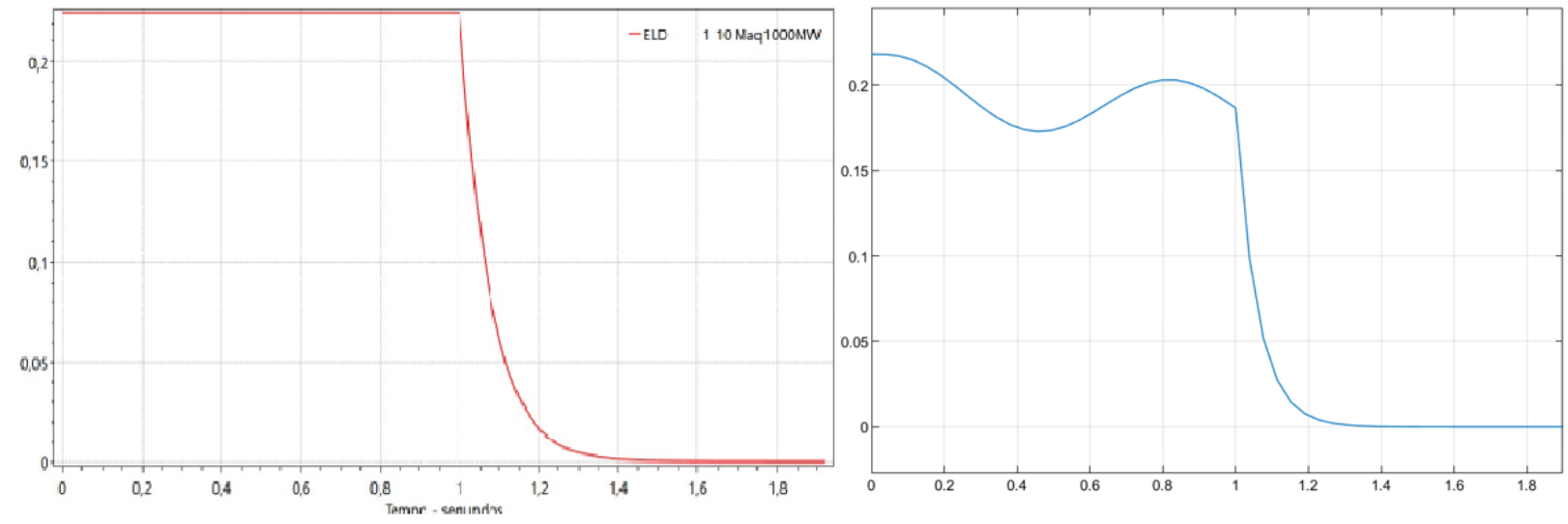

FIgURA 9. Tensão interna transitória de eixo direto $\left(E_{d}^{\prime}\right)$. (Autoria própria) 
Na figura 10 a tensão interna ao eixo de quadratura, diminui de forma linear após a falta tendendo a zero, assim como a tensão do eixo direto.
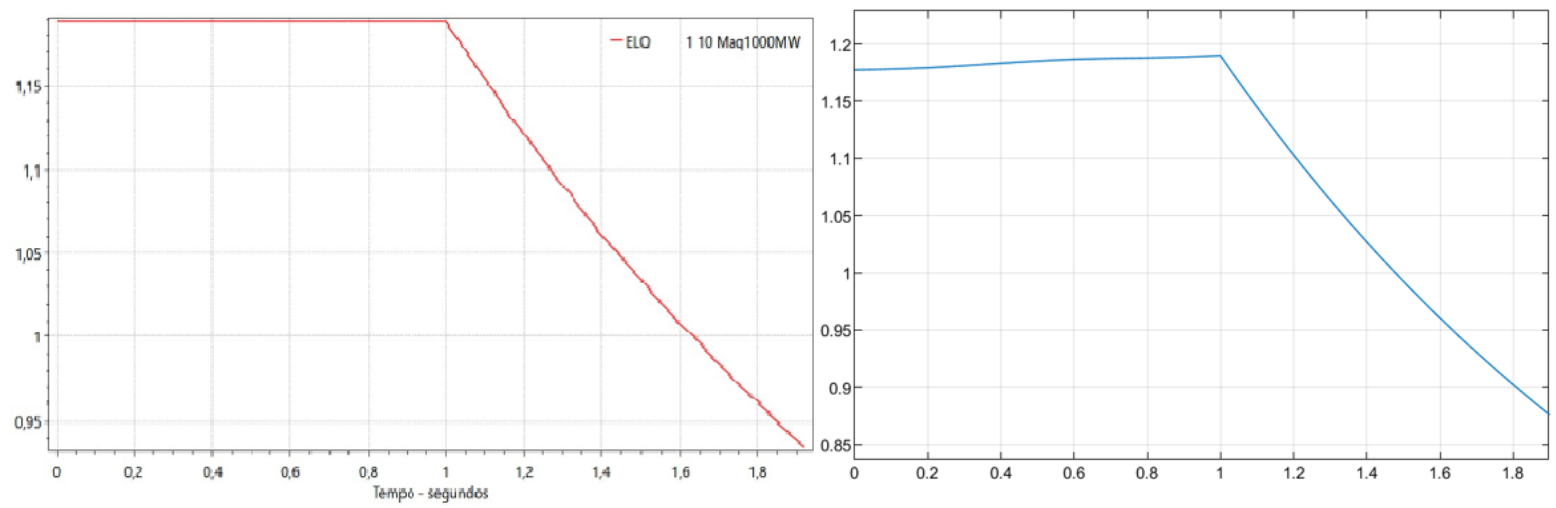

FIGURA 10. Tensão interna transitória de eixo de quadratura $\left(E_{q}^{\prime}\right)$. (Autoria própria)

Na figura 11 é apresentado o efeito da tensão terminal no eixo de quadratura e no eixo direto, os gráficos evidenciam o comportamento da equação (4), onde a soma das tensões deve ser igual a zero.

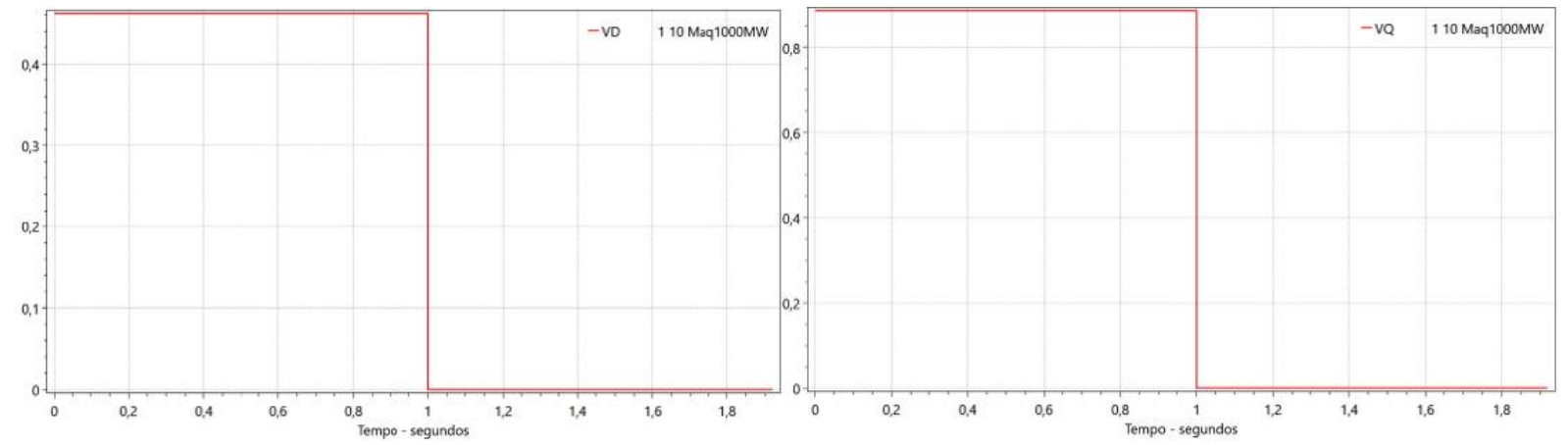

FIGURA 11. Tensão terminal do eixo de quadratura e eixo direto. (Autoria própria)

\section{CONCLUSÕES}

O principal objetivo desse trabalho é obtido ao mostrar que os resultados obtidos utilizando o modelo de dois eixos implementado ao Simulink ${ }^{\circledR}$ são satisfatórios representando bem o comportamento do resultado do ANATEM. É notório que o ANATEM não leva em consideração o balanço natural da máquina síncrona em uma situação de regime permanente. Por consequência, antes de 1 segundo o regime permanente é mostrado sem oscilação das grandezas da máquina. Posteriormente, as simulações, foi verificado que, para este intervalo de duração do curto, o sistema manteve o sincronismo. Oscilou e encontrou um novo ponto de operação.

\section{AGRADECIMENTOS}

Ao professor Doutor Adriano Aron Freitas de Moura, pela orientação, apoio e confiança. Diante do tema proposto, sempre esteve presente e soube indicar a direção correta que o trabalho deveria tomar. Obrigado por me manter motivado durante todo o processo. Também quero agradecer á Universidade Federal Rural do Sémiárido e o seu corpo docente que demonstrou estar comprometido com a qualidade e excelência do ensino. 


\section{REFERÊNCIAS}

[1] STEVENSON, W. D. J. Elementos de Análise de Sistemas de Potência. [s.1.] McGraw-Hill, 1974.

[2] C.G. da MATA - "Uma contribuição na análise da estabilidade transitória dos sistemas elétricos de distribuição na presença de geração distribuida”, Dissertação de Mestrado em Engenharia elétrica, Brasil, agosto de 2005.

[3] COSTA, A. J. A. S.; SILVA, A. S. E. Controle e Estabilidade de Sistemas Elétricos de Potência: apostila. Florianópolis: Universidade Federal de Santa Catarina, 2000.

[4] BOLDEA, I. Variable Speed Generators. 1st ed. Boca Raton: Taylor \& Francis Group, 2006.

[5] HOMRICH, Guilherme. Métodos para determinação de parâmetros de máquinas síncronas de imãs permanentes. 2016. 155 f. Tese (Doutorado) - Curso de Pós-Graduação em Engenharia Elétrica, Ufrs, Porto Alegre, 2016.

[6] CENTRO DE PESQUISAS DE ENERGIA ELÉTRICA. Manual do usuário do programa de análise de transitórios eletromecânicos - ANATEM: Versão 11.02.00, 2017.

[7] SAUER, Peter W; PAI, M. A.; CHOW, Joe H.. Power System Dynamics and Stability: With Synchrophasor Measurement and Power System Toolbox. 2. ed. New Delhi, India: Ieee Wiley, 2018

[8] RODRIGUES, Tiago Granato. Análise de Curto Circuito em um Gerador Síncrono de Pólos Salientes pelo Método dos Elementos Finitos. 2013. 82 f. Tese (Doutorado) - Curso de Engenharia Elétrica, Universidade Federal do Rio de Janeiro, Rio de Janeiro, 2013.

[9] COELHO, João Paulo; PINHO, Tatiana. Introdução ao Simulink®: Aplicações na engenharia. Bragança Paulista: Ipb, 2015.

[10] SADAAT, Hadi. Power Systems Analysis. 2. ed. New York: Mc Graw Hill, 2002. 712 p. 